\title{
The Architecture of MOOCs Platform Based on Virtual Technology
}

\author{
Jinjuan Quan
}

Tianjin University of Finance and Economics, Tianjin, China, 300222

qjj_16@163.com

Keywords: MOOCs; Virtual technology; VMware; VMware sphere

\begin{abstract}
MOOCs platform is an important part of MOOCs construction and promotion. This paper describes the problems that exist in the current MOOCs platforms and propose a solution which is based on Virtual technology. By use of VMware server component and function, the architecture of MOOCs platform is construct and this platform improve the usage of hardware. The combination of Virtualization and MOOCs platform improved the effectiveness of the MOOCs platform resources, extensibility and stability greatly.
\end{abstract}

\section{Introduction}

MOOCs is called Massive Open Online Courses. In the fall of 2011, Stanford professors Peter Norving and Sebastian Thrun filmed the lectures form their artificial intelligence course and put the videos online. They opened registration to anyone, anywhere in the world. That attracted hundreds of thousands of students. More than 16000 students signed up and of the 248 students who received perfect scores, everyone took the course online, rather than at Stanford. The success of the Stanford AI course made MOOCs------Massive Open Online Courses------front page news. It also leads to the launch of Coursera and Udacity. At the same time, an MIT and Harvard venture evolved into edX as a non-profit consortium for online education. Both groups have now grown substantially [1, 2].

MOOCs are also received much attention In China, According to the data of Coursera, there are 13million Chinese users registered in 2013 and ranked ninth in the world wide. The number reached 65 million in 2014 and the growth rate is far more than other countries. The Tsinghua University, Peking University has cooperated with edX launch the MOOCs. At the same time, Fudan University Shanghai Jiaotong University joined in Coursera launch their own MOOCs. There is a budding revolution in global online higher education. It has become increasingly convenient that the learners use terminal equipment via network to complete course independent. More and more university provide free course for global learners. As long as learners are interested in the course, they can learn any course after registered regardless of nationality, skin color and age $[3,4]$.

Nothing has more potential to lift more people out of poverty-by providing them an affordable education to get a job or improve in the job they have. Nothing has more potential to unlock a billion more brains to solve the world's biggest problems. And nothing has more potential to enable us to reimaging higher education than the massive open online course, or MOOCs.

MOOCs platform, as an important part of MOOCs, since the date of birth, shouldering the sharing of resources to achieve quality education, promote the reform of higher education, dissemination of outstanding achievements of civilization, promote learning society formed, contribute to the country's economic development and promote overall social progress $[5,6]$.

The uses of MOOCs platform include student registration and certification, courses video management, course test management, interaction between teacher and students or students between students management, generating charts based on big data analysis and other functions. Rational use of MOOCs platform plays a key role in MOOCs construction, application and promotion $[7,8,9]$.

\section{Problems}

MOOC is massive open online course, the numbers of users online at the same and the operating scale cannot be compared to traditional courses. If there are One hundred and sixty thousand 
students study, discuss and evaluate on line at the same time, the results are unthinkable. The problems that hinder the development of MOOCs platform are as follows:

1) Many students login and study at the same time will lead to momentary overload.

2) With the increase of the number of users, the amount of hardware will also increase and the cost of investment will be overlarge.

3) The MOOCs servers are composed of server clusters, some of them are responsible for user registration, and some are responsible for store the videos and online group discussion, and some are responsible for big data analysis evaluation.

\section{Solutions}

Virtualization Technology. With the emergence of virtualization technology, Virtual Machine, which including operating systems and applications appeared. There are a lot of virtual machines running on a single server and each of them is isolated. The virtualization software layer can separate the virtual machines from the host and allocate the resource according to demand dynamically. Without changing the operating system and application, the virtualized MOOCs platform server can accomplish the task with the virtual hardware. The hardware is isolated with software and every machine can run lots of virtual machine and deployment to run multiple applications [10].

VMware Server Components and Functions. This MOOCs platform use the VMware virtualization solutions VMware vSphere, it includes interface layer, virtualization layer and management layer, etc. As shown in Fig. 1 [11].

The interface layer includes vSphere Client, vSphere Web Client and other components. It is mainly responsible for connecting and accessing between the users and vCenter Server.

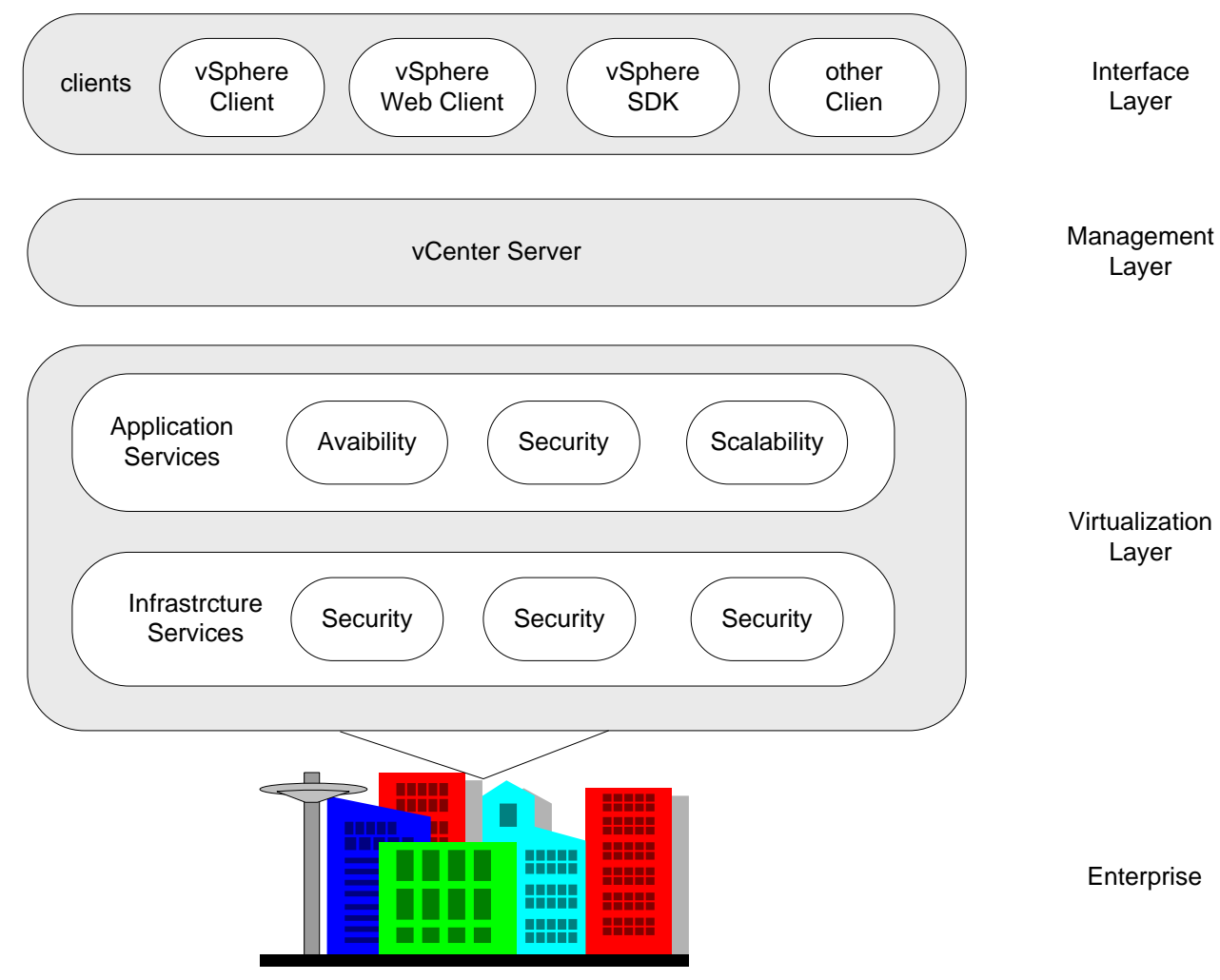

Figure 1. VMware Server Components and Functions

The virtualization layer consists of architecture service layer and application service layer. The architecture service layer is MOOCs platform private cloud, including the server's CPU, storage, switches and network. This layer provides main hardware and resource for virtualization and virtual 
resource pool. Application service layer consists of the application software. It services to ensure the availability, security and scalability of virtual machine software and services. This layer is mainly composed of virtual machine software, VMware ESX and ESXi server. VMware vSphere 5.5 is based on ESXi hypervisor architecture. It is not build dependent on the operating system as simplified virtualization management program. The ESX architecture is console operating system and has been a new version of ESXi replaced.

Core of management layer is VMware vCenter Server, which is the core of the architecture of vSphere. It implements for VMware ESX and ESXi servers centralized management, configuration and management of virtualized information environments. It provides services in data center access control, performance monitoring and alert management and etc.

In routine maintenance of the MOOCs platform, many advanced features must be accomplished by the vSphere Client or Web Client to log in to vCenter Server management interface to operate. Such as vMotion (virtual machine live migration), vDS (dynamically allocates and balances computing capacity), HA (failure to restart the virtual machine) and other functions.

The server's CPU, memory, storage and networking resources can be virtualized to multiple virtual hosts. Through effective manage and schedule of VMware ESX and vCenter Server, by collecting different hardware resources to form a unified logical resource pools. Multiple applications can run simultaneously. Utilization of resources is improved greatly.

VMware Server System Architecture of MOOCs Platform. MOOCs platform VMware server virtualization system consists of Virtual center management server, Virtual Manufacturing, ESX / ESXi which are two architecture of vSphere, VI client (vSphere Client), Web browser, graphics terminals, FC switch, IP networks, server farms, FC storage array, iSCSI(internet small computer system interface) storage arrays, NAS (network access server) storage array and the backup servers. As shown in Fig. 2.

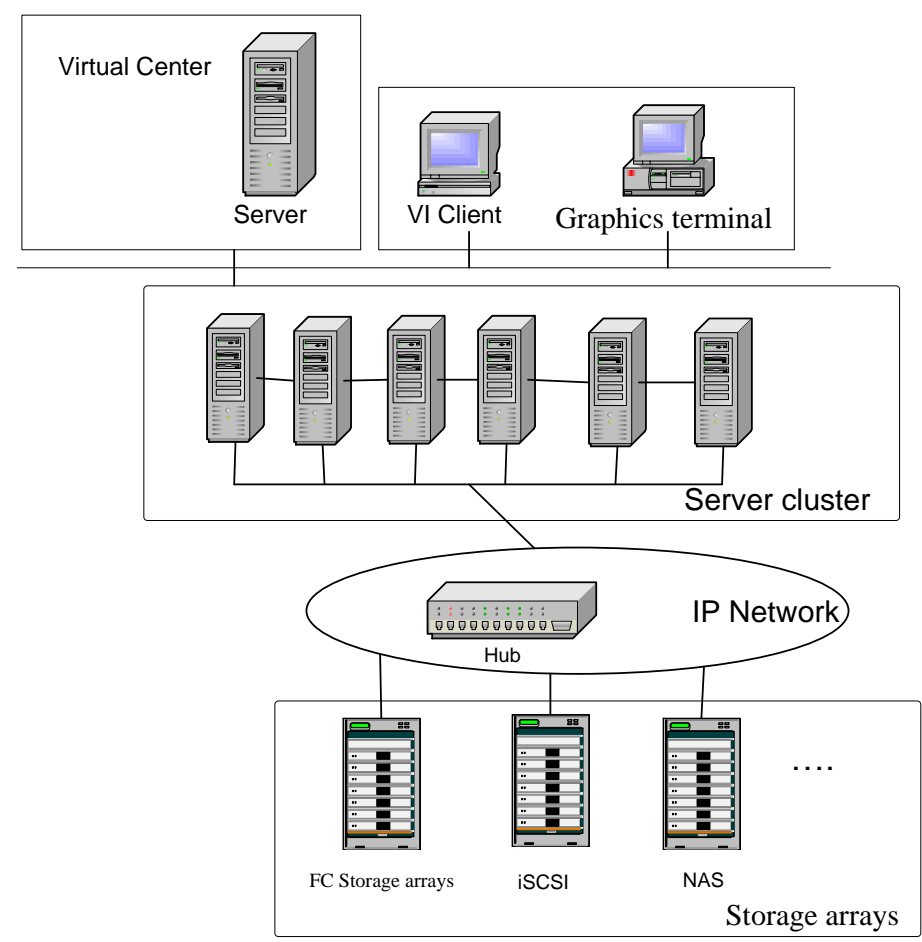

Figure 2. Architecture of the MOOCs platform VMware server

VI Client (vSphere Client), Web browser and graphics terminal is the user access end of access layer, which connect to vCenter Server to complete centralized management of ESXi server configuration and management of virtualized information environment.

Server cluster connected to a shared storage array FC storage (Fibre Channel storage array), iSCSI (Internet Small Computer System Interface) storage arrays, NAS (Network Access Server) storage array through the fiber switch or IP network, thus completing the MOOC platform private cloud structures. 
The private cloud of the MOOC platform virtualizes CPU, memory, network, storage into many VM virtual machines, which are installed in the ESXi server groups.

Through effective management and scheduling of vCenter Server, different hardware resources form a unified logical resource pools. The MOOC platform can provide registration, video learning, classroom discussions and data analysis at the same time. The utilization of resources is improved greatly.

If the MOOC server contracts the virus or operating system breakdown, the Backup server via High Availability of VMware vSphere to restart the affected virtual machine and FT function immediately active a secondary copy of the virtual machine to provide continuous service.

\section{Summary}

This paper presents a MOOCs platform solution which is based on Virtual technology. By use of VMware server component and function, the architecture of MOOCs platform is construct and this platform improve the usage of hardware.

The advanced features of VMware such as VMware vSphere virtualization vMotion, HA and FT solved the problems that the server upgrade. The combination of Virtualization and MOOCs platform improved the effectiveness of the MOOCs platform resources, extensibility and stability greatly.

\section{References}

[1] Ayala, Cesar; Dick, Geoffrey, Treadway and Jayson. The MOOCs are Coming! Revolution or Fad in the Business School?[J]Communications of the Association for Information Systems: Vol. 35(2014),p.225-243

[2] Rosé, Carolyn P., Goldman, Pam, and Lauren .Supportive technologies for group discussion in MOOCs [J].Current Issues in Emerging eLearning: Vol. 2(2015): Iss. 1, Article 5.

[3] Kong Yi. Uses of Cloud Computing in Laboratory Construction and Management of University and Colleges[J].China Education Technoloay \&Equipment.Vol.12(2013),p.28-31

[4] Sadiku, M.N.O, Musa, S.M., Momoh, O.D. Cloud Computing: Opportunities and Challenges Potentials, IEEE Vol.33 (2014), p.34 - 36

[5] Stanoevska-Slabeva K, Wozniak T. Cloud Basics-An Introduction to Cloud Computing [J].Grid and Cloud Computing, vol.1 (2010), p.47-61

[6] Deimann, Markus.The dark side of the MOOC - A critical inquiry on their claims and realities, Current Issues in EmergingeLearning,Vol. 2: Iss. 1, Article3.

[7] Macfadyen, L., \& Dawson, S. Numbers Are Not Enough. Why e-Learning Analytics Failed to Inform an Institutional Strategic Plan. Educational Technology \& Society, Vol. 15(2012), p. 149-163.

[8] P.Y.Thomas. Cloud computing.A potential paradigm for practising the scholarship of teaching and learning $[\mathrm{J}]$, The Electronic Library vol.29(2011), p.214-224

[9] Armando Fox. Cloud computing in education [EB/OL]. http://inews.berkeley. edu/articles/Spring2009/cloud computing

[10] Singh MA, Shrivastava M. Overview of Security issues in Cloud Computing [J]. International Journal of Advanced Computer Research vol.2 (2012) p.41-45.

[11]ZHANG chen-xiang, ZHU Dan-chen:Construction Plan Optimization for College Computer Laboratories Based on Cloud Computing[J].Journal of Huaiyin Institute of Technology Vol.22(2013), p.28-30 